\section{MS5-P45 Structure and mechanism studies of human tyrosinase}

Xuelei Lai ${ }^{1,2}$, Montserrat Soler-López ${ }^{2}$, Harry J. Wichers ${ }^{3}$, Bauke W. Dijkstra ${ }^{1}$

1. Laboratory of Biophysical Chemistry, University of Groningen, Groningen, The Netherlands

2. ESRF-The European Synchrotron

3. Wageningen University and Research Center, Wageningen, The Netherlands

\section{email: lai@esrf.fr}

Tyrosinase (EC 1.14.18.1) is a binuclear copper-containing enzyme that is widely distributed throughout bacteria, fungi, plants and animals. The enzyme catalyzes the $o$-hydroxylation of monophenols to the corresponding $o$-diphenols and the subsequent conversion of the $o$-diphenols to the corresponding $o$-quinones. Tyrosinases have been implicated in a variety of biological functions. In plants, tyrosinases have been suggested to participate in wound healing, defense reactions and in the synthesis of flower pigments. In fungi, tyrosinases have been postulated to participate in spore formation, defense reactions, and pigmentation. In mammals, including humans, the enzyme is responsible for skin pigmentation abnormalities, such as albinism, vitiligo and other melanin-related syndromes.

Human tyrosinase is a type 1 trans-membrane glycoenzyme that is found specifically in neural crest-derived pigment-producing cells (melanocytes) of the skin, choroid and iris and in the neuroectoderm-derived RPE (Retinal Pigment Epithelium) of the eye. It catalyzes the initial and rate-limiting steps of melanin pigment production. Mutations in the human tyrosinase gene directly cause oculocutaneous albinism type 1 (OCA1), an autosomal recessive disorder characterized by reduced melanin pigment in the hair, skin and eyes.

The aim of this project is to solve the crystal structure of human tyrosinase and its substrate/inhibitor bound structures using crystallography, and characterize some of the most novel mutants that cause OCA1. These information will help us to understand the structure basis for the oculocutaneous albinism type 1 and provide valuable information for drug design. We have successfully expressed human tyrosinase in insect cells as secreted protein and is fully active. We also crystallized it under several different conditions and the crystals diffracted up to 4 Angstrom in the first diffraction test.

\section{MS5-P46 Helicobacter pylori protein function assignment through the 3D structure}

\author{
Giuseppe Zanotti ${ }^{1}$
}

1. Department of Biomedical Sciences, University of Padua, Via Ugo Bassi 58/B, 35131 Padua, Italy

email: giuseppe.zanotti@unipd.it

Despite the identification of $H$. pylori dating back to 1984 , its pathogenesis remains poorly understood at the molecular level. In our laboratory we are working on the structural characterization of proteins of the bacterium relevant for pathogenesis or host colonization. In this communication we will focus on four secreted proteins, whose function was unknown or undefined, and we will show how the crystal structure, combined with other experimental evidences, can provide clues about the physiological function of the protein.

HP1561. Its high-resolution structure reveals a bi-lobed fold with a narrow cleft between the $\mathrm{N}$ - and C-terminal domains. CeuE shares a common architecture typical of Class III periplasmic binding proteins. Crystal structure and solution data demonstrate that it binds $\mathrm{Ni}^{2+}$ through a metallophore.

HP1028. It is a protein relevant for colonization and for the survival of the bacterium in the stomach. The three-dimensional structure of the protein reveals that it belongs to the family of lipocalins, a group of proteins that bind and transport small molecules. Its structure, along with the localization of the mature protein in the bacterial periplasm and the position of $h p 1028$ gene in the bacterial genome, points to a role in $H$. pylori chemotaxis.

HP1029. This protein has a sequence identity (around $35 \%$ ) with a putative $\beta$-D-galactosidase and with members of the YhcH/YjgK/YiaL protein family. Its structure is very similar to that of the only member of the DUF386m family whose three-dimensional structure is known, YhcH from $H$. influenza, but a $\mathrm{Zn}^{2+}$ ion is present in the active site.

HP1454. It is a protein of 303 amino acids found in the extracellular milieu. Its structure presents an elongated bent shape, composed by three distinct domains that possess folds already present in other structures. The crystal structure does not allow a clear identification of the function.

Keywords: Helicobacter pylori; pathogens; gastritis

Keywords: enzyme, crystallography 